\title{
Shock wave attenuation in aqueous foam
}

\author{
L. Domergue, R. Nicolas, J.-C. Marle, L. Matthey, M. D'aloisio, \\ L. Buche \& C. Hubert \\ CEA-DAM Isle de France, BP12, 91680 Bruyères-le-Châtel, France
}

\begin{abstract}
The capacity of aqueous foam to decrease the pressure produced by explosive material in close proximity areas is well known. This paper presents some results of shock wave attenuation obtained in aqueous foam during explosive experiments. We conducted our experiments in two steps: Firstly, a spherical device of $6.6 \mathrm{~kg}$ of Plastrite (pentrite based explosive substance) was held at a height of 2 meters and initiated at its centre in an open air range. The evolution of pressure was measured by means of seven PCB pressure pencil gauges at the same height, and at various ranges from 2 to 5 meters. Secondly, a wide structure was filled with aqueous foam at an average density of 1:125 and the same test was performed inside the foam. The results of those tests are discussed in terms of the overpressure, Time of Arrival and blast wave velocity in each case. The results are compared to relevant literature at every step. Furthermore, the results between the two tests are compared to reach a conclusion on the capacity of aqueous foam required to decrease blast effects on a structure.

Keywords: shock wave attenuation, foam capacity, overpressure, velocity of shock wave.
\end{abstract}

\section{Introduction}

It is well know that aqueous foam can decrease the effect of a shock wave caused by an explosive device. In this way, we have performed two different experiments in our explosive area. The first one was performed in air and the second one was performed in foam with a density of 1:125. This paper presents some results of overpressure measurements obtained from the detonation of a sphere made with $6.6 \mathrm{~kg}$ of Plastrite ${ }^{\circledR}$ (pentrite base explosive material). In both cases, the measurements of pressure were carried out to allow a comparison of pressure, due to explosions in air and in the foam. Seven PCB pencil gauges 
were used for the tests. We firstly present in this paper our experimental device. Secondly, we present the results obtained in air and we compare them to the laws proposed by Kinney and Graham [1]. Thirdly, we present the results obtained in foam and we compare them to literature laws proposed by Sandia laboratories [2]. Finally, we compare the results of overpressures and average speeds of blast waves in air and foam, before concluding that foam is a good media to dramatically decrease the effect of shock waves caused by detonation for structures as well as for humans.

\section{Background}

The foam's capacity to decrease blast effects has already been investigated by Sandia National Laboratories [2] 20 years ago, by using pencil pressure gauges. They performed tests at several foam densities and several weights of $\mathrm{C} 4$ explosive. An analytical formulation of the overpressure level and Time of Arrival has been proposed empirically. Furthermore, Kinney and Graham [1] published more than 20 years ago their "Explosions in air". The comparison with their theory allows us to ensure that the way we choose to perform blast wave characteristics measurements is accurate enough and produced good results.

\section{Experimental settlements}

We have carried out our tests with seven PCB pencil gauges. The types of sensor are free field blast pencil gauges with integral electronics. They have the advantage of being perfectly usable in explosive experiments in open range areas, by allowing long cable driving. We have chosen two different types of gauges. The first one is able to measure overpressure from 0 to 3.4 bars and for the distance closer to the explosive device; we used gauges with a 0 to 34 bars capacity range.

We know that the theory of shock wave propagation in air is based on some assumptions:

1. detonation occurs in a continuous and non finite area without any obstacle;

2. the explosive charge is a spherical one and the ignition occurs in its centre;

3. the air is considered to be a perfect gas;

4. the detonation is considered to be isentropic.

To keep our experiments close to the theory, we have chosen to control the few parameters below:

1. the explosive material is hung at the height of $2 \mathrm{~m}$ from the ground;

2. the explosive material is placed in a spherical envelope, with a view to approaching the spherical geometry $(+/-2 \mathrm{~cm})$, and the detonator is close to the centre of the sphere;

3. the gauges are placed at the same height as the device to decrease the risks to measure reflected waves from the ground;

4. the measurements were taken far enough away from the fireball to allow us to consider that detonation occurs in air and to avoid perturbations in electrical signals. 
The picture presented in figure 1 shows the experiment configuration in air and the placement of the gauges.

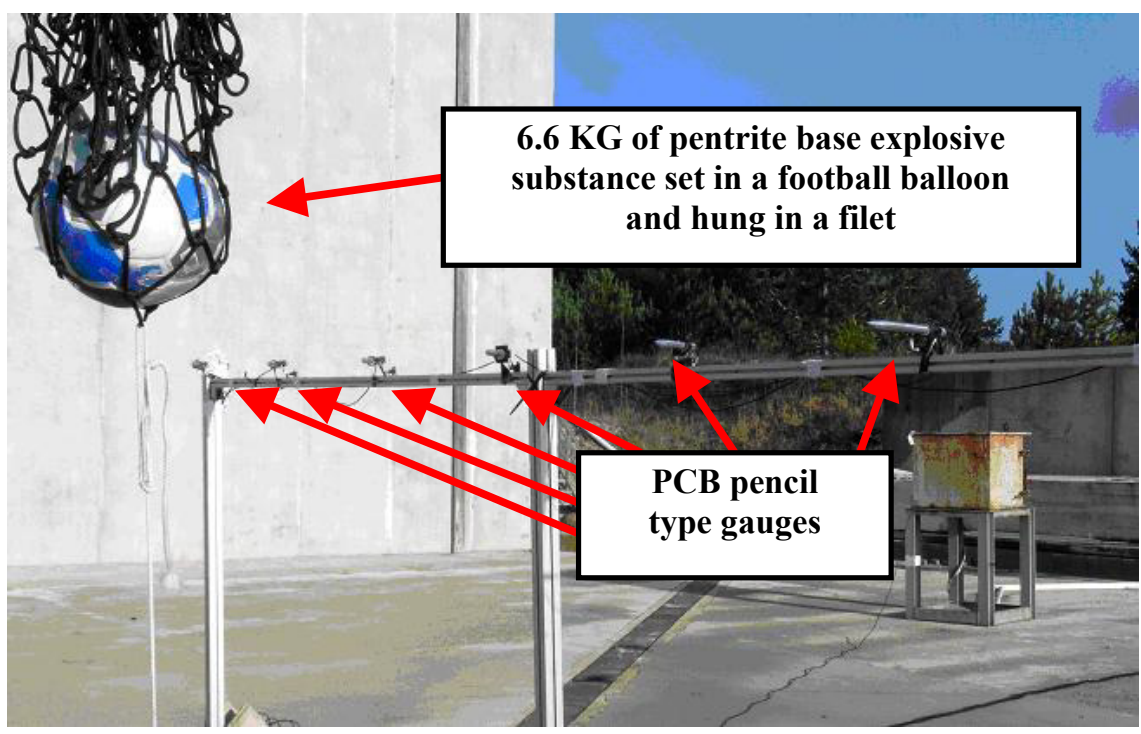

Figure 1: $\quad$ Picture of experiment in air with pencil type gauges.

\section{Experimental results in air and comparison to bibliography}

Figure 2 presents an example of signals obtained in air. One can remark that the curves present a double top due to wave reflection by the ground.

The main values of our experiments in air are summarized in table 1. One can notice that the duration of the positive blast wave is quite difficult to measure, due to the shape of the signal that represents the characteristics of a ground reflection.

\subsection{Comparison between explosive materials}

Abaca and analytics data are available for the TNT, which is the reference explosive material. For the Plastrite explosive material, we have used an equivalent weight factor of 1.27 to calculate the pressure, in order to compare the result with bibliography sources. This means that the pressure due to a mass of $1 \mathrm{~kg}$ of Plastrite at a given distance is the same (in similar experimental conditions) to the pressure obtained with $1.27 \mathrm{~kg}$ of TNT. For our experiments, the $6.6 \mathrm{~kg}$ of Plastrite has the same behaviour as that of $8.3 \mathrm{~kg}$ of TNT.

\subsection{Comparison between overpressures in air}

The values for pressure, the Time of Arrival and the wave average velocity are found in literature with scaled values. We also use the scale distance to allow us 
to compare our results with Kinney [1]. The following data are also expressed in this way:

$$
r=R / W_{e}^{1 / 3}
$$

where $r$ is the scale distance; $R$ is the distance in meters and $W_{e}$ is the explosive weight of TNT equivalent. Those hypotheses allow us to present the graphic in figure 3 below, which describes the evolution of overpressure versus the distance from the centre of the explosive device. Furthermore, in the second part of this paper, we assume that those rules are still valid for foam, which will allow us to compare our results with the literature from the Sandia laboratory.

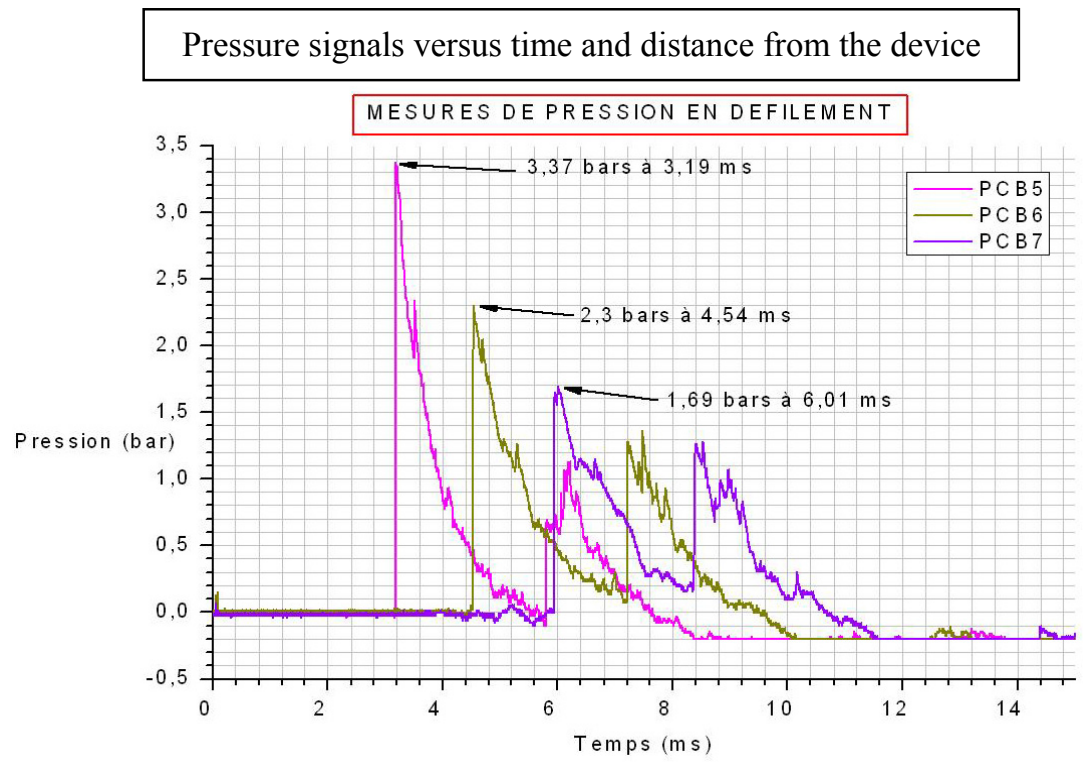

Figure 2: $\quad$ Signals obtained in air with PCB gauges number 5, 6 and 7.

Table 1: $\quad$ Main results of our experiment in air.

\begin{tabular}{|c|c|c|c|c|}
\hline Gauge \# & $\begin{array}{c}R \\
\text { Radial Distance to } \\
\text { the charge centre } \\
(\mathrm{m})\end{array}$ & $\begin{array}{c}P_{+} \\
\text {Overpressure } \\
(\text { bar })\end{array}$ & $\begin{array}{c}t_{a} \\
\text { Time of } \\
\text { Arrival }(\mathrm{ms})\end{array}$ & $\begin{array}{c}U=R / t_{a} \\
\text { Average velocity } \\
\text { of shock wave } \\
(\mathrm{m} / \mathrm{s})\end{array}$ \\
\hline PCB 1 & 2.03 & 9.62 & 1.04 & 1933 \\
\hline PCB 2 & 2.12 & 9.09 & 1.16 & 1834 \\
\hline PCB 3 & 2.50 & 7.26 & 1.52 & 1653 \\
\hline PCB 4 & 3.00 & 4.55 & 2.16 & 1394 \\
\hline PCB 5 & 3.65 & 2.53 & 3.19 & 1150 \\
\hline PCB 6 & 4.38 & 1.74 & 4.54 & 969 \\
\hline PCB 7 & 5.10 & 1.31 & 6.01 & 859 \\
\hline
\end{tabular}




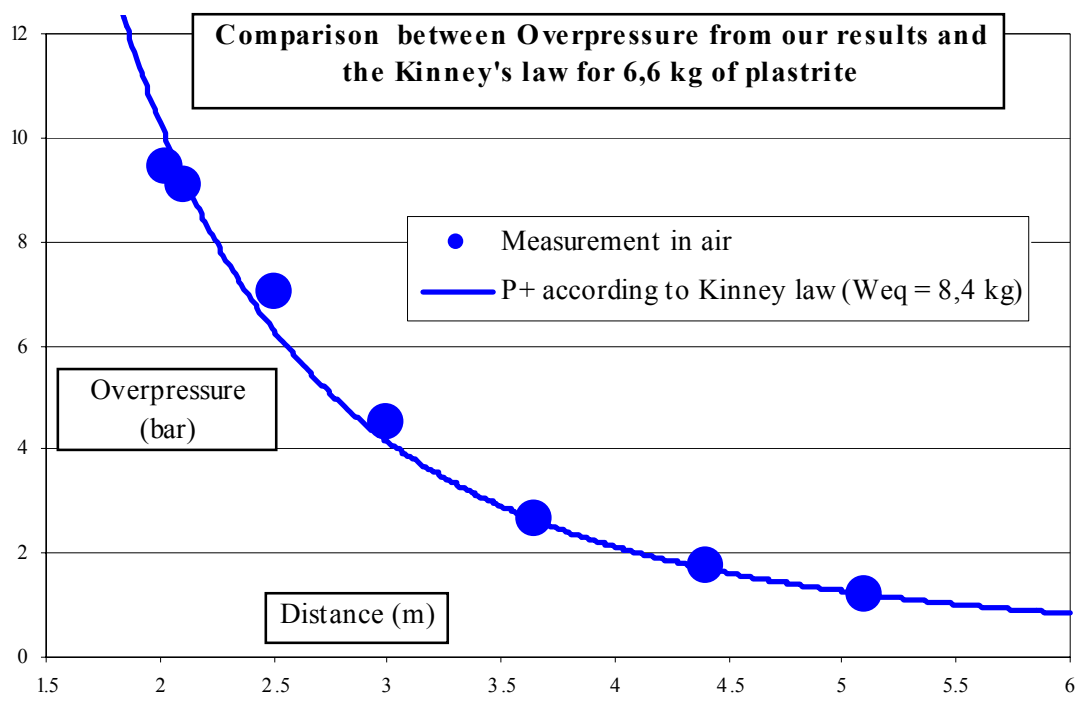

Figure 3: Overpressure comparison between measurements and Kinney's law.

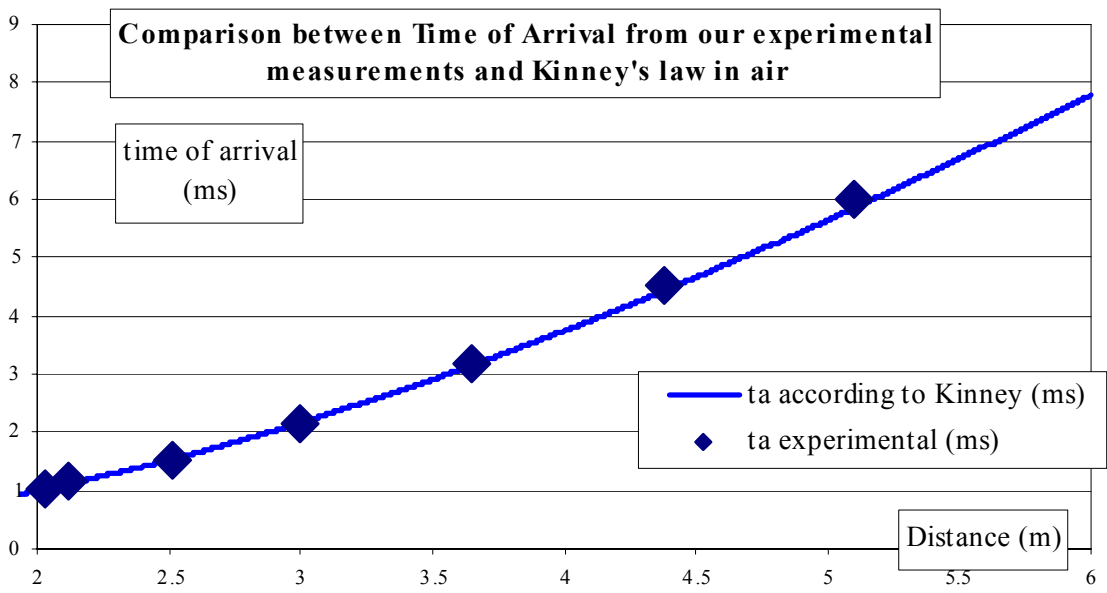

Figure 4: Time of Arrival comparison between measurements and Kinney.

\subsection{Comparison between Times of Arrival in air}

The Time of Arrival measurements can be achieved thanks to the fact that all gauges have been synchronized with the precise time of detonation. The front of the pressure is so sharp that it allows us to determine the Time of Arrival of the blast wave in air accurately. Figure 4 shows a good agreement between Kinney's law and our results. 


\subsection{Comparison between the average speeds of the blast wave in air}

The calculation of the average speeds of the blast wave is achieved through the determination of the Time of Arrival. The average speed $U$ at the distance (or radius) $R$ is calculated simply as follows:

$$
U(R)=t_{a} / R
$$

The values of the average speeds presented in figure 5 below are also compared with Kinney [2].

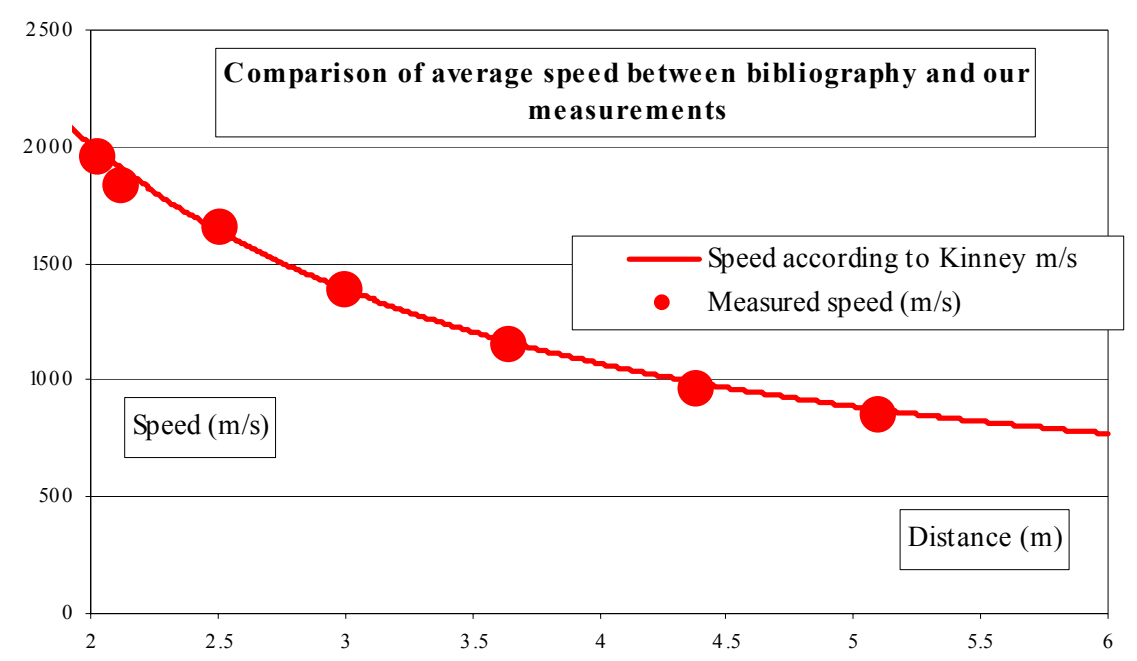

Figure 5: Comparison of speed between our measurements and Kinney's law.

One can see a good correspondence between our results and the bibliography, as well as for the overpressure, Time of Arrival and average speed. We have also decided to perform the tests in foam with a good chance of success by using the same experimental process. The next section presents the results we obtained with foam of 1:125 density.

\section{Experimental results in foam and comparison to literature}

In order to perform the experiments in foam, we used the same gauges as in air with the same methodology and we carefully avoid foam (or moisture) penetration inside the connections. The graph in figure 6 presents an example of signals from pencil PCB gauges in foam.

Although the gauges are entirely in aqueous foam, the quality of signals is good. The main values from our experiment in foam are summarized in table 2 . 


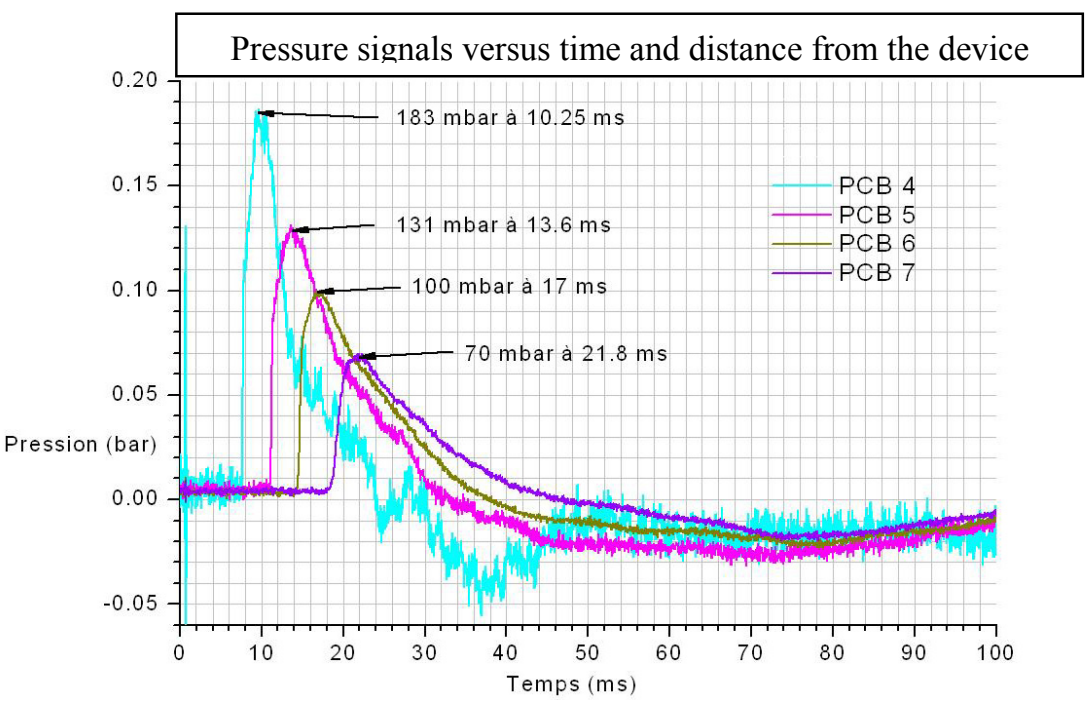

Figure 6: $\quad$ Signals acquired with PCB gauges 4, 5, 6 and 7.

Table 2: $\quad$ Main results of our experiment in air.

\begin{tabular}{|c|c|c|c|c|}
\hline Gauge & $\begin{array}{c}R: \\
\text { Radial Distance } \\
\text { to the charge } \\
\text { centre }(\mathrm{m})\end{array}$ & $\begin{array}{c}P_{+}: \\
\text {Overpressure } \\
(\text { bar })\end{array}$ & $\begin{array}{c}t_{a}: \\
\text { Time of } \\
\text { Arrival }(\mathrm{ms})\end{array}$ & $\begin{array}{c}U=R / t_{a} \\
\text { Average velocity } \\
\text { of shock wave } \\
(\mathrm{m} / \mathrm{s})\end{array}$ \\
\hline PCB 1 & 2,10 & 1.85 & 2.5 & 800 \\
\hline PCB 2 & 2.25 & 0.88 & 3.27 & 780 \\
\hline PCB 3 & 2.65 & 0.53 & 4.85 & 525 \\
\hline PCB 4 & 3.24 & 0.18 & 7.20 & 435 \\
\hline PCB 5 & 3.90 & 0.13 & 10.58 & 360 \\
\hline PCB 6 & 4.55 & 0.10 & 14.00 & 325 \\
\hline PCB 7 & 5.20 & 0.07 & 18.00 & 288 \\
\hline
\end{tabular}

\subsection{Comparison between explosive materials}

Sandia laboratory used C4 for their experiments. So, to compare the Sandia published empirical laws with our results, we need to consider an equivalent factor of pressure between $\mathrm{C} 4$ and pentrite. We calculated that this factor should be approximately 1.08 .

\subsection{Comparison between overpressures in foam}

As one can see in figure 7, we reach a good agreement between our experimental results and Sandia laws in foam although one can remark that a difference for the closer gauge from the device exists. 


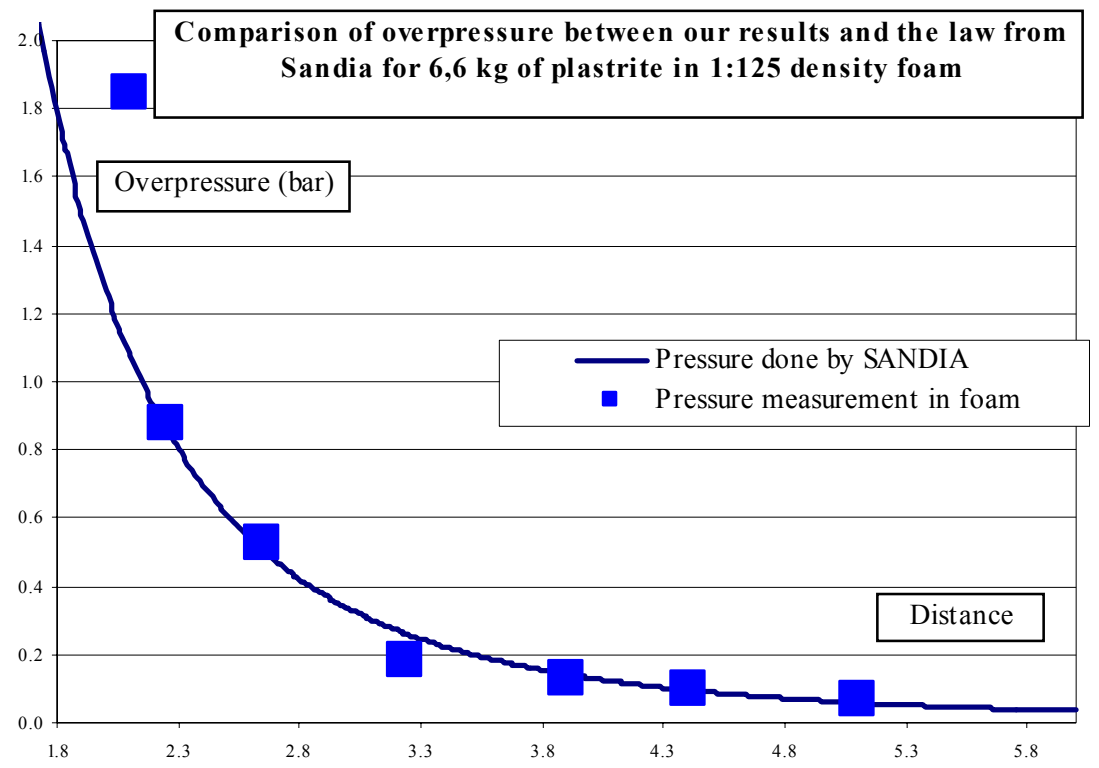

Figure 7: Overpressure comparison between the measure and the Sandia law.

\subsection{Comparison between the Times of Arrival in foam}

This measurement is collected with the same methodology as used in air. All gauges have been synchronized with the instant of the time of detonation. Nevertheless, the front of pressure is less sharp than in air, as one can see in the example of signals shown in figure 6 . So the Times of Arrival are more difficult to determine. The graph presented in figure 8 shows some differences between our results and the Sandia law. Those differences may come from the properties of the foam used to perform our test. The fact that the Time of Arrival is greater than was seen in the literature could indicate that our foam reduces the velocity of the blast wave more effectively.

\subsection{Comparison of overpressure results between air and foam}

Figure 9 shows the comparison between the evolution of pressure in air and in foam with a density of 1:125. As one can see, the capacity of foam to decrease shock wave overpressures is particularly efficient. This allows one to choose foam with a view to protecting structures from blasts.

\subsection{Comparison of the average speeds between results in air and foam}

Our experiments show the decrease of average speeds more than doubled between the experiments in air and in foam. Furthermore, the average velocity 
decreases more rapidly in our foam than in the experiments performed by Sandia. The way to make the foam and its type are factors to take into account when trying to understand why.

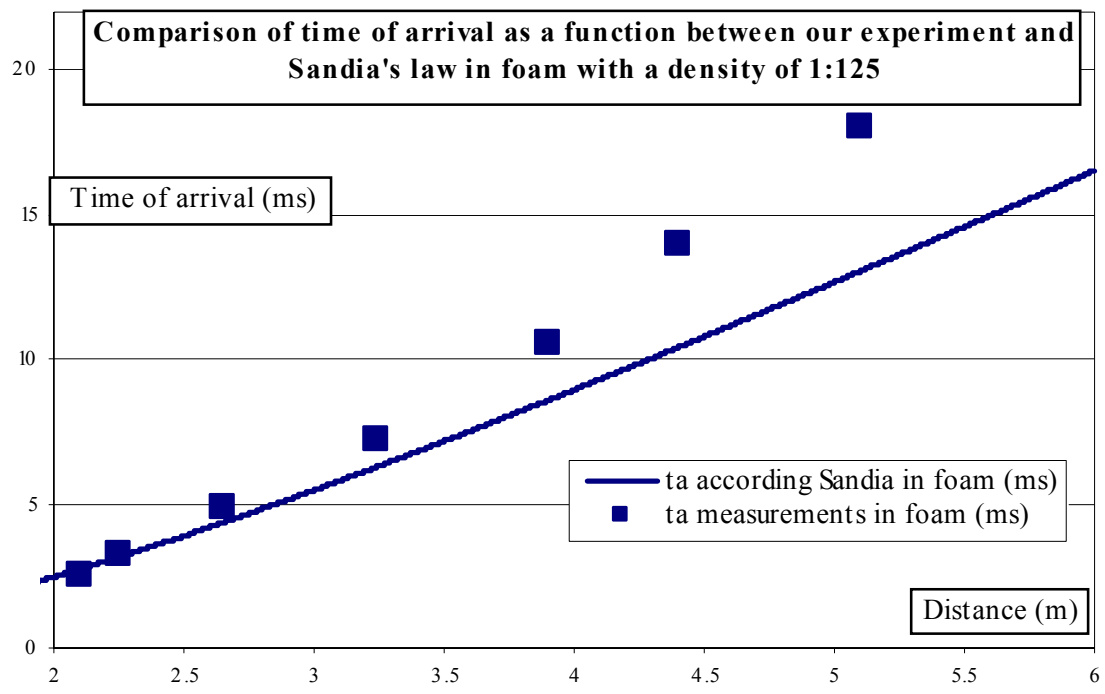

Figure 8: Comparison between Times of Arrival in foam.

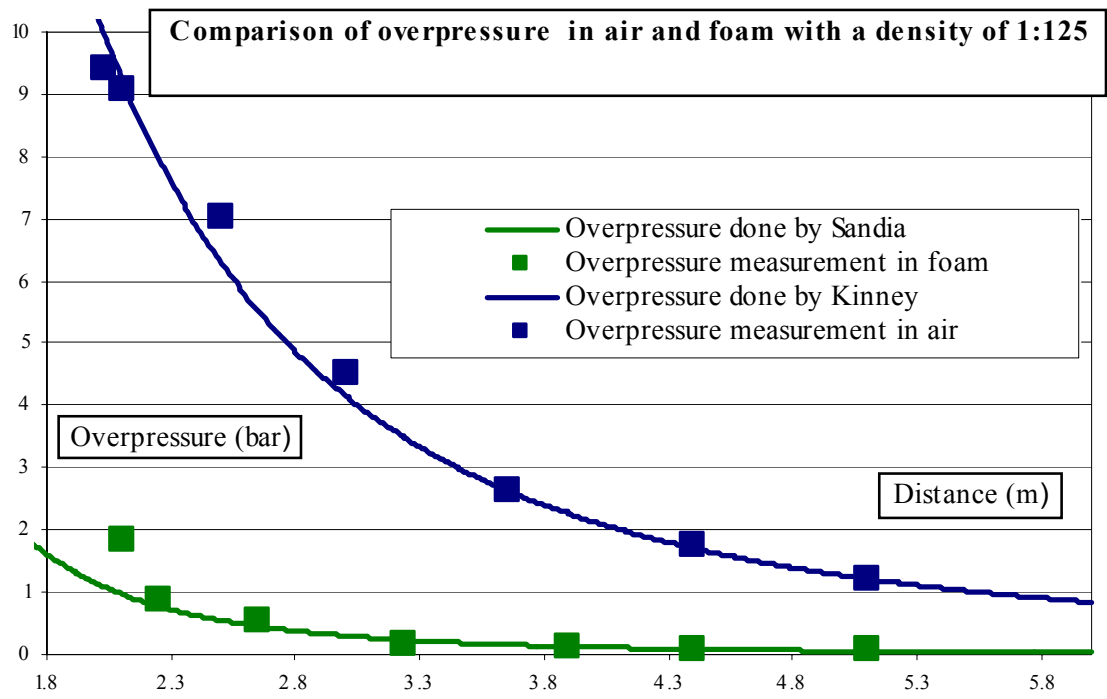

Figure 9: Comparison between overpressure in air and foam. 


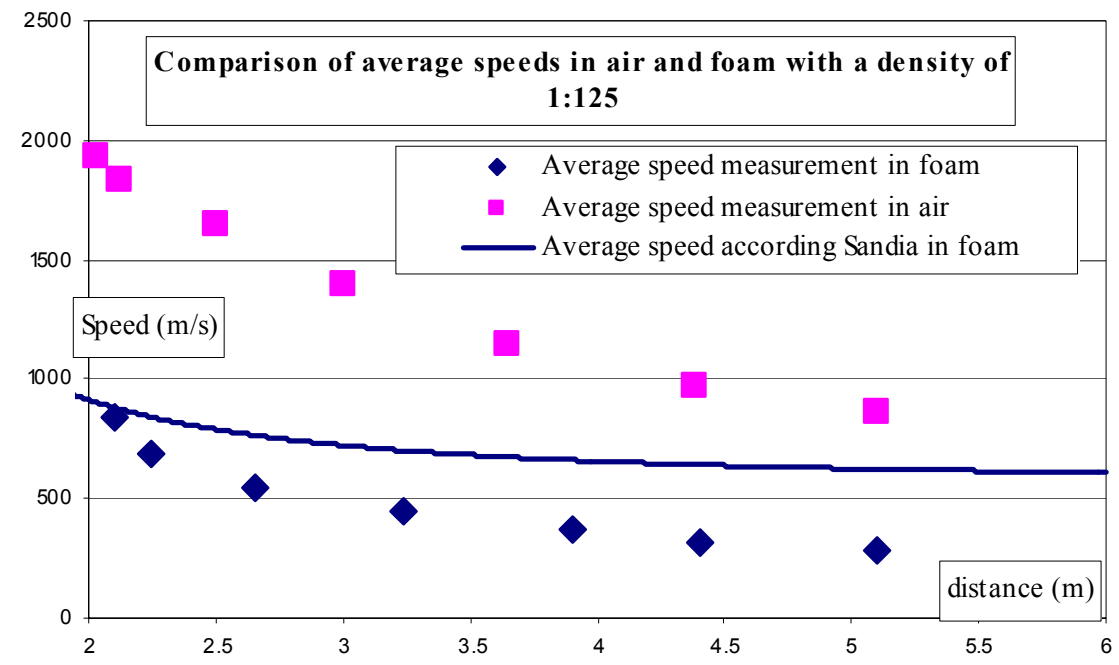

Figure 10: Comparison of average speeds in air and foam.

\section{Conclusions}

This paper shows the capacity of aqueous foam to dramatically decrease the overpressure shock wave by a factor of 5 to 10 . Furthermore, we found a decrease in the average speed of shock waves of more than a factor of 2 . A second step would be to measure and to compare impulsion between several types of explosives material. Furthermore, some other experiments should be done with different foam densities with a view to comparing the shock absorption capacity. The aim could be to determine the capacity of foam to protect human lives as well as the integrity of structures from blasts, which could occur by accident or maliciously.

\section{References}

[1] G.F. Kinney \& K.J. Graham "Explosions in air" Springer-Verlag 1985.

[2] F. William "Blast Mitigation Capabilities of Aqueous Foam" Sandia National Laboratories - report N 0533, February 2006. 